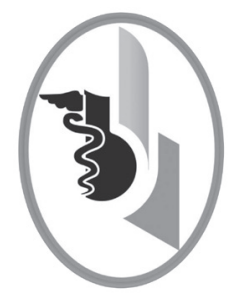

\title{
UN CASO DE EVISCERACIÓN DE PELVIS POR CARCINOMA COLOIDE DEL RECTO ${ }^{*}$
}

\author{
A CASE ABOUT PELVIC EXENTERATION BY MUCINOUS \\ RECTAL ADENOCARCINOMA
}

Espinosa-París Hernando ${ }^{1}$

Espinosa-Dorothy J.2

\section{RESUMEN}

En el año 1953 fue publicado un documento de la autoría de un importante profesor en el campo de la cirugía general que marcó escuela dentro de la Facultad de Medicina de la Universidad de Cartagena-Colombia en la segunda mitad del siglo XX. El doctor Hernando Espinosa París, nació en la capital de la República de Colombia, realizó estudios médicos en la Universidad Nacional de Colombia y especialización en Cirugía en los Estados Unidos. Realizó importantes aportes en el ámbito médico cartagenero, en lo asistencial y en su labor como docente. Estos aportes también contribuyeron al desarrollo de la cirugía, en una época en que los especialistas se preciaban de adelantar grandes y radicales intervenciones operatorias para abordar enfermedades, como los cánceres en diversos contextos anatómicos. Las fotografías usadas en este documento hacen parte de la Fototeca Histórica de la Medicina Cartagenera, del proyecto de investigación adelantado por el semillero de investigación HISTORIMED, de la Universidad de Cartagena-Colombia. Rev.cienc.biomed. 2015;6(2):406-415.

PALABRAS CLAVE

Cirugía; Cáncer; Intestino Grueso; Tubo digestivo.

\section{SUMMARY}

A document written by an importan professor in the area of general surgery was published in 1953, this professor played an important role in medicine department in the Universidad de Cartagena- Colombia in the second half of twentieth century. Hernando Espinoza París was born in Colombia, he studied medicine in the Universidad Nacional de Colombia and then, he made a specialization in surgery in the United States of America. He made important contributions in the medical field of Cartagena, both assistance and the professor, this contributions also improved the surgery area, at a time where the specialist carried out radical interventions to address diseases like cancer in various anatomical contexts. The pictures used in this document are part of the Fototeca Histórica de la Medicina Cartagenera, (investigation project by semillero de investigación HISTORIMED from the Universidad de Cartagena-Colombia). Rev.cienc. biomed. 2015;6(2):406-415

\section{KEYWORDS}

Surgery; Cancer; Large intestine; Gastrointestinal tract.

(*) Reproducido textualmente de la Revista de la Facultad de Medicina de la Universidad de Cartagena. Volumen II. Números 3-4. Marzo-abril. 1953.

1 Médico. Profesor de Cirugía de la Facultad de Medicina de la Universidad de Cartagena y cirujano del Hospital Sanatorio San Pablo. Cartagena. Colombia.

2 Enfermera Graduada. 


\section{NOTA DEL EDITOR}

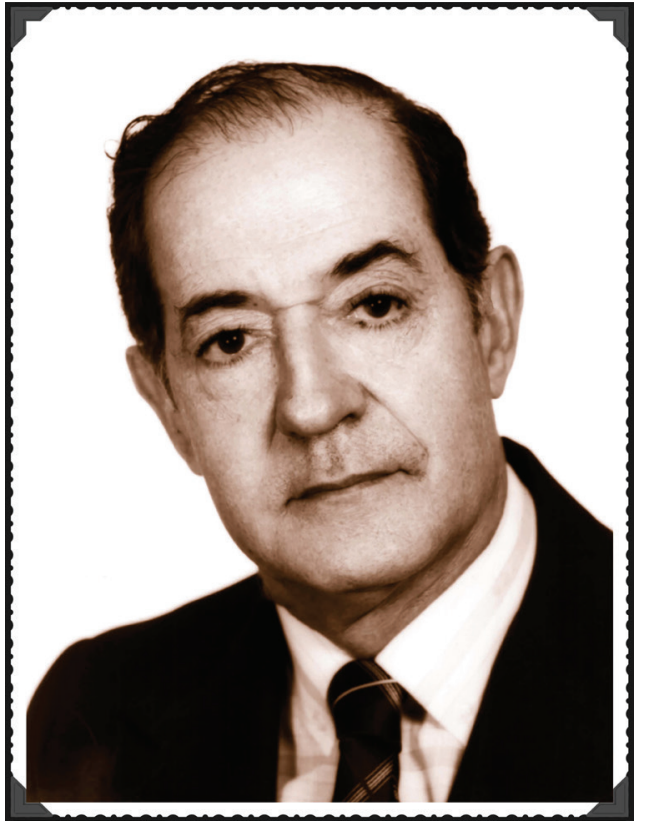

Hernando Espinosa París

El profesor Hernando Espinosa París fue docente de Cirugía de la Facultad de Medicina de la Universidad de Cartagena en la segunda mitad del siglo XX. Fue jefe de cirugía del Hospital Sanatorio San Pablo en CartagenaColombia, donde realizó numerosas cirugías para el manejo de las secuelas de la tuberculosis. Poseía gran destreza quirúrgica y amplio manejo de la anatomía, por lo cual abordó con éxito diversas patologías que comprometían distintos tejidos o territorios anatómicos.

En el ámbito colombiano fue reconocido por su labor quirúrgica, docente y empresarial en el área de la medicina y también como ferviente defensor de los conceptos de la ética médica. Temprano para su tiempo, con enorme valor personal y profesional, con visión constructiva y crítica escribió el documento titulado "Iatrogenia", el cual presentó como ponencia en varios congresos médicos. El contenido fue publicado inicialmente en la Revista Colombiana de Gastroenterología en 1989, reproducido por otras revistas científicas y comentado en la prensa general y médica.

También escribió otro documento que denominó "Algunos conocidos aforismos en ciru-

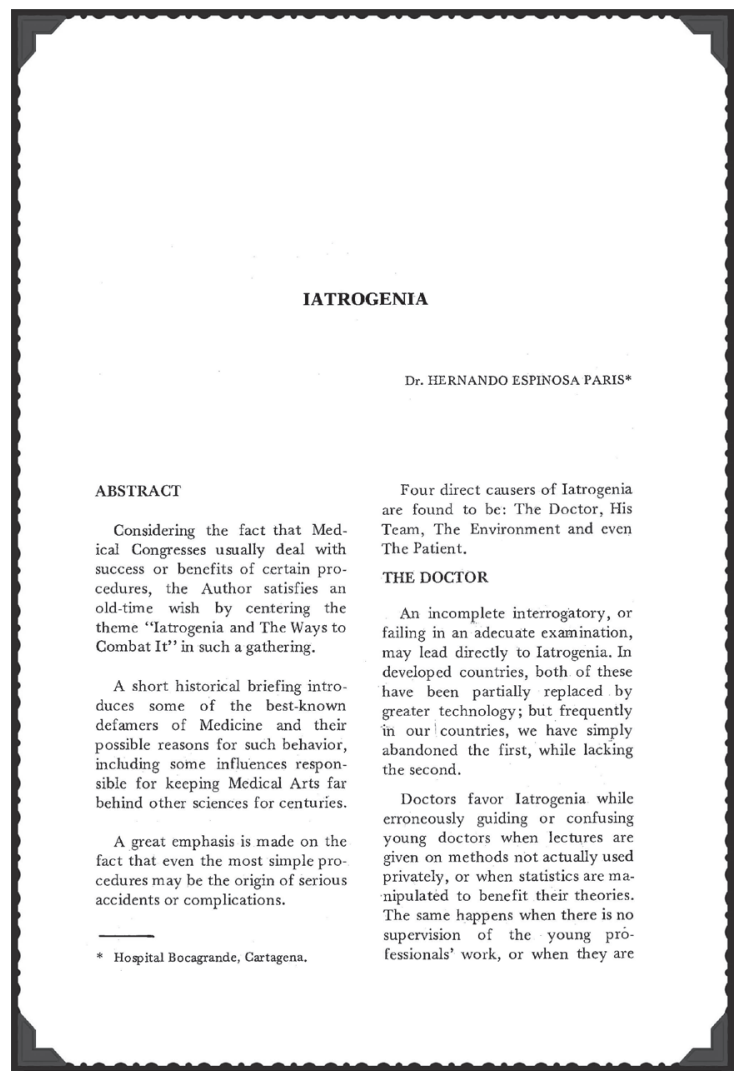

gía biliar que vale la pena recordar", dirigido a los médicos y cirujanos en formación. Escribió varios artículos científicos que fueron publicados en la revista de la Facultad de Medicina de la Universidad de Cartagena. Estudió medicina en la Universidad Nacional de Colombia en Bogotá y realizó especialización en cirugía en New York University Postgraduate Medical Scohool.

Al regresar a Colombia fijó su residencia en Cartagena y desempeñó una importante labor docente, administrativa y asistencial, sobre todo esta última. En esta ocasión, Revista Ciencias Biomédicas reproduce el contenido de una de sus lecciones impartidas en el servicio de cirugía de la Facultad de Medicina de la Universidad de CartagenaColombia, a partir de un caso clínico que atendió e intervino. Por la fecha de publicación, puede ser posiblemente su primera publicación; dicho sea de paso, en este documento aparece como coautora su esposa Dorothy J. Espinosa quien era enfermera graduada. El artículo fue finalizado con 15 referencias bibliográficas las cuales no son reproducidas. 


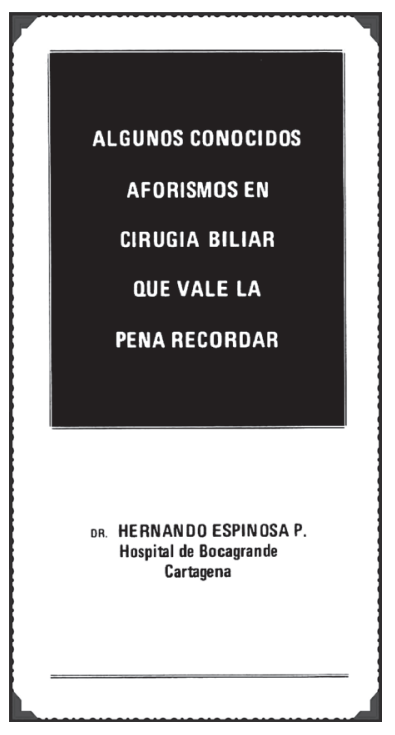

CONSIDERACIONES GENERALES

Parece casi increíble que en nuestro medio estrecho, puedan los pacientes, y seguramente que lo hacen, acostumbrarse a los grandes cambios que en su anatomía y en su funcionamiento corporal significan una colostomía permanente, o la implantación de los uréteres a la piel del abdomen. Sin embargo, en los pocos casos en que he tenido la oportunidad de tratar personalmente, los pacientes se muestran resignados, y lo que pudiéramos llamar tan conformes o aun contentos, como no lo imaginaban sus familiares, ni menos los cirujanos que han ocasionado esas necesarias modificaciones anatómicas.

Tal es el caso de la paciente que presentamos en este estudio, a quien se le practicó una evisceración completa de la pelvis y que está retornando a su vida normal en forma rápida y sorprendente.

Es mi opinión personal, que esos tipos de intervenciones deben ser explicadas más o menos en detalle a los miembros responsables de la familia, para tener la autorización de practicar la cirugía mutilante que requieren variedades de cáncer; y solamente dar una idea vaga y no muy precisa a los pacientes pues por lo común rehusarían en el principio y no la aceptarían hasta tanto su enfermedad no se hiciera apremiante en extremo, y con ello como cualquiera lo puede comprender, resultaría una pérdida de tiempo precioso e irreparable, y seguramente cuando regresaran a someterse a las operaciones diagnósticas o es muy tarde para ellos, o estas que ser mucho más amplias y menos prometedoras de lo que hubieran sido tempranamente.

Es algo que sorprende verdaderamente, el hecho de que exámenes tan sencillos y que no requieren demasiada especialización o alta técnica, como son un tacto rectal o el uso de un proctoscopio sean tan poco usados por médicos y cirujanos en todas partes del mundo y posible e infortunadamente con mayor frecuencia entre nosotros.

Si pensamos en un momento en que los tumores malignos de la terminación del tubo digestivo, son las más fáciles de diagnosticar a un examen médico completo y detallado, y que son los mayores índices de operabilidad y supervivencia dan, veremos la tremenda responsabilidad que un profesional contrae con el paciente que ha confiado en la minuciosidad de esos exámenes su tranquilidad y su salud.

Revisando las estadísticas norteamericanas, leemos que las dos terceras partes de los cánceres de colon están colocados en el recto sigmoides, y que de ellos $75 \%$ son diagnosticables con el dedo, y $90 \%$ con la ayuda de proctoscopio. Es decir, es un tipo de cáncer en que el médico general se halla capacitado para diagnosticar en 9 veces de 10, a la primera consulta, y sin emplear los exámenes complementarios que se necesitan para otros casos, como por ejemplo cáncer de estómago, riñón, columna vertebral, etc., que requieren muchas veces el concepto del médico especialista y complicados métodos de laboratorio. En síntesis creemos que el diagnóstico de los cánceres del recto y sigmoides concierne a los médicos generales de la misma manera que a los especialistas, es decir es algo semejante a la relativa facilidad con que un médico general usualmente diagnostica fibromatosis uterina o un quiste del ovario.

Al contemplar los desalentadores resultados de los canceres del estómago y páncreas por ejemplo, la dificultad en su diagnóstico precoz, su baja resectabilidad, un poco más de una cuarta parte en los del estómago contra $90 \%$ de los del recto y sigmoides en las grandes clínicas, y la supervivencia de 5 años tan baja, $2 \%$ en los del estómago, $0 \%$ en los del páncreas, contra casi $50 \%$ en los del recto y sigmoides, debemos entusiasmarnos más donde las condiciones son más favorables, facilidad en el diagnóstico y mejor respuesta del organismo a los tratamientos y hacer los exámenes completos a todos los pacientes 
que nos llegan a la consulta, tanto a los cirujanos y especialistas.

\section{INCIDENCIA}

Los cánceres de colon, ocupan con frecuencia, el segundo lugar en el hombre y el cuarto en la mujer; de ellos $80 \%$ están localizados en el sigmoides y el recto, de 75 a $78 \%$ son palpables y $90 \%$ visibles al sigmoidoscopio y según algunos autores por los menos el $75 \%$ están desarrollados a expensas de adenomas. En una serie de Duke sobre 1000 cánceres del recto sigmoides, encuentra que 650 son de hombres y 350 de mujeres. De $80 \%$ a $90 \%$ se presentan en pacientes por encima de la cuarta década de la vida.

\section{ETIOLOGÍA}

No se sabe exactamente cuántos cánceres del recto y sigmoides se han formado en un pólipo preexistente, pues cuando consultan el crecimiento del tumor ha hecho desparecer el pólipo, pero se calcula que ese porcentaje es alto, según algunos $40 \%$. En todo caso la formación de carcinomas con polipósis múltiple preexistente es alta, Hullsieck en 128 caos de polipósis encuentra que el $46 \%$ desarrollaron posteriormente carcinomas. También parece haber cierta relación, aunque más baja que la anterior, entre colitis ulcerativa crónica, principalmente de los jóvenes y desarrollo posterior de carcinomas.

\section{PATOLOGÍA}

De los cánceres del recto y sigmoides, 95\% son de tipo glandular y son los llamados adenocarcinomas, y $3.5 \%$ de tipo epitelial. Menos frecuentes son los sarcomas que suelen ser particularmente malignos y los melanomas.

Los cánceres del recto y sigmoides dan metástasis por tres vías. Contigüidad o infiltración local, linfática y hematógena. La primera es la más común de las formas de extensión, y la fijación a los órganos vecinos, indica muy frecuentemente extensión del tumor, aunque esta fijación puede estar ayudada por la infección usualmente concomitante, viéndose este fenómeno con mayor frecuencia en los cánceres de la porción final del tubo digestivo que en los del resto del colon. La extensión anterior es la más frecuente, para el hombre la vejiga y las vesículas seminales, rara o muy tardíamente a la próstata, pues parece que la aponeurosis de Denonvillier es una buena barrera de defensa; en la mujer a la pared vaginal posterior y útero, menos frecuentemente a la vejiga. La extensión posterior hacia el sacro es solamente de los casos avanzados. Estas extensiones se hacen más por invasión que por verdadera metástasis. Las otras vías, linfáticas y hematógenas es sabido que se hacen en forma relativamente tardía. Los cánceres del recto y sigmoides son de los del intestino grueso los que más metástasis dan al hígado, pleura y riñones. Suderland ha encontrado que el $27 \%$ dan metástasis hematógenas.

Respecto al pronóstico en los operados, de los cánceres del tubo digestivo son de los que más esperanzas dan, ya que según las grandes clínicas como la Lahey, el 90\% de los operados no tienen recurrencia los cinco años en los casos confinados solamente a la pared del recto sin intervención de los tejidos vecinos, ganglios regionales o vasos sanguíneos. El $37 \%$ en aquellos con ganglios solamente positivos, $30 \%$ en los que tienen ganglios y tejidos vecinos; y solamente $14 \%$ en los que hay presencia de células tumorales en los vasos.

Respecto a la localización de los tumores del recto y sigmoides, hay actualmente una tendencia a clasificarlos por encima o por debajo de la reflexión anterior del peritoneo, a la que se halla colocada a unos de los 8 a 10 centímetros del ano.

La razón más importante para esta clasificación consiste en que el pronóstico de los cánceres colocados por debajo es notablemente peor que el de los colocados por encima. Algunos autores les dan término de peritoneales a los unos y extraperitonales a los otros. En general los tumores de los sigmoides y del recto disminuyen la supervivencia a medida que se acercan al ano.

Gultbert Dukes de Londres hace una clasificación según la extensión de los tumores en tres grupos: Grado A: el tumor se halla limitado solamente a la pared del recto, se es- 
peran 5 años de supervivencia en el $91.5 \%$ de los casos. Grado B: diseminación de los tejidos extrarectales, sobrevivencia en $64 \%$. Grado C: metástasis a los ganglios regionales, $16 \%$. Según el Memorial de New York, este $16 \%$ sube en sus manos al $39.4 \%$.

\section{SINTOMATOLOGÍA}

Los cánceres del recto y sigmoides pueden considerarse como bastante silenciosos y su presencia es revelada frecuentemente mas por sus complicaciones que por ellos mismos. De las complicaciones la más frecuente es la ulceración, y con esta la presencia de sangre, moco y pus en las deposiciones. La ulceración se presenta más comúnmente en los carcinomas de tipo nodular o coloide, y menos en los de tipo papilar o en los sarcomas.

En general pues, el síntoma más frecuente y posiblemente el primero anotado por los pacientes es la presencia de sangre macroscópica y roja en las deposiciones, puede decirse que la casi totalidad de los pacientes lo presentan y suelen consultar por ello. No debe omitirse una completa revisión del recto en personas quejándose de sangre en las deposiciones y con la presencia de hemorroides, pues según la Clínica Mayo el 15\% de los cancerosos del recto han sido recientemente hemorroidectomizados, y ese porcentaje sube a $25 \%$ según las estadísticas del Mount Sinai de New York.

Después de la presencia de la sangre siguen en frecuencia la presencia de moco y pus en cantidades variables en las deposiciones; luego los cambios en los hábitos en la defecación de los pacientes, siendo a veces este el primer síntoma presentado pero que había pasado más o menos desapercibido y solo es descubierto al interrogatorio cuidadoso. Estos cambios suelen ser, estreñimiento, o bien diarreas o la combinación de las dos, sensación de defecación incompleta. Cambio de forma de bolo fecal; dolores difusos abdominales; anemia; pérdida de peso; aumento en la velocidad de sedimentación; dificultades en la micción.

Se han agrupado estos síntomas en cuatro grandes grupos así: 1. Deposiciones anorma- les: presencia de sangre, moco o pus; cambio de forma en el bolo fecal. 2. Cambio en los hábitos de los movimientos intestinales: constipación, diarrea o la combinación de las dos. 3. Molestias y dolores abdominales, sensibilidad, sensación de peso y llenura del recto, impresión de defecación incompleta o defecación dolorosa. 4. Presencia del tumor, anemia y caquexia en los casos avanzados, lo mismo que dificultad en el funcionamiento de la vejiga.

\section{TRATAMIENTO}

Está casi completamente reservado a la cirugía, con sus resecciones amplias, de las cuales hay varios tipos. Para los cánceres de sigmoides y alto recto, pueden practicarse la extirpación local del tumor y la anastomosis, siempre que se puedan dejar por lo menos 6 centímetros de tejido sano por debajo del límite del tumor, aunque algunos cirujanos rebajan esta distancia en algunos centímetros basados en el hecho de que los cánceres tienden a difundirse considerablemente más hacia arriba que hacia abajo.

Para los tumores más bajos situados, la resección abdominoperineal, Ilamada comúnmente operación de Miles, con disección abdominopélvica de los ganglios es la operación de elección, y que suele dar los alentadores resultados que hemos considerado anteriormente. Algunos autores recientemente prolongan la disección de los ganglios a todo lo largo de la arteria mesentérica inferior ligándola muy cerca de la aorta y extirpando los ganglios y las cadenas ganglionares desde debajo de la tercera porción del duodeno. Más recientemente Black de la Mayo Clinic 1952 ha publicado los resultados de la resección abdominoendorectal, en 41 casos, con muy satisfactorios resultados, siendo este procedimiento en varios tiempos principalmente en la parte abdominal muy semejante a la operación de Miles, la parte más difícil consiste en la preservación de la irrigación de la parte distal que ha de continuar funcionando; la parte endorectal se hace en vez de la perineal, y como su nombre lo indica se practica a través de los esfínteres dilatados, resecándose el tumor que ha sido previamente aislado del resto del intestino por ligaduras, la conservación del canal da 
un buen porcentaje de excelente funcionamiento esfinteriano pues no todos los casos lo dan, debido al largo periodo en que debe estar ampliamente dilatado el canal anal con la parte redundante de intestino a través de él ya que no se usan suturas entre los cabos, sino aplicación directa y resección del sobrante a las dos semanas. Personalmente no tenemos la menor experiencia con este tipo de resección, pero indudablemente que es algo nuevo y atrayente.

Cuando el tumor está situado tan bajo que invade al ano, es recomendable la disección bilateral de los ganglios inguinales como complemento de la operación de Miles.

\section{PRE Y POSTOPERATORIOS}

No quiero detallar cuidadosamente ni hacer mayor énfasis en la importancia de la preparación tanto del estado general del paciente que se va a operar del intestino mismo, pues es cosa sabida por todos que son problemas claves para el buen éxito de las intervenciones.

Encuentro de una gran comodidad la incisión transversal amplia, con sección de los rectos abdominales, que da una ancha vía de acceso a todas las partes de la cavidad pélvica y a la mayor parte del abdomen, además facilita la colostomía en el ángulo izquierdo sin ser necesario practicar nuevas incisiones y economía en el tiempo necesario para ellos.

Una vez abierto el abdomen practicar un cuidadoso examen de todos sus órganos, principalmente del hígado, pues numerosas metástasis en él, contraindicarían la intervención.

El uso durante la operación y en el inmediato postoperatorio de los catéteres de polietileno es una innegable ventaja en los trabajos de cirugía que requieren grandes cantidades de fluidos, principalmente en nuestros medios, donde los cirujanos no siempre disponen del personal humano tan bien adiestrado como en las grandes clínicas americanas. La introducción del catéter en la vena da al cirujano de seguridad de una franca vía para la administración de fluidos intravenosos, principalmente sangre, en forma rápida si es necesario, sin el peligro de las "perdidas de la venas" a las infiltraciones en los momentos de mayor urgencia durante las intervenciones.

Nosotros los hemos usado en varias ocasiones en quemaduras extensas o intervenciones de mayor envergadura, empleando las venas de las extremidades y siguiendo el método de la deserción y ligadura de la vena, cortándola por encima de esta e introduciendo el catéter a unos cuantos centímetros y fijándolo con dos ligaduras suaves a las venas. Haciendo después una mínima contraventura en la piel un centímetro por debajo de la incisión, se saca por ella el catéter y fija con esparadrapos y se cubre con apósitos, de tal manera que cualquier movimiento que haga el paciente o sea necesario hacerle no ocasionará la salida del tubo y se tendrá la seguridad permanente con una vía cómoda para la administración de fluidos en cualquier momento. Pudiendo permanecer este tubo por varios días sin que se oblitere, se le evita en el postoperatorio al paciente los sufrimientos que implican las punciones venenosas y el cansancio de la quietud de los brazos durante las aplicaciones de las soluciones glucosas etc. Hemos notado que cuando usamos terramicina a través de catéter, la región se inflama y la vena se pone dolorosa.

Los cuidados postoperatorios de las resecciones abdominoperineales son los que atañen a las grandes intervenciones y como bien sabemos hoy día en unión de los del preoperatorio y la intervención misma, forman un gran conjunto al que se le deben los éxitos de la cirugía moderna.

Nos detendremos solamente a considerar los cuidados referentes a las colostomías, las irrigaciones perineales y más adelante a los de las implantaciones a la piel del abdomen de los dos uréteres. En los casos en que la resección se limita solamente a recto sigmoides el tipo de colostomía es llamado secos, en que aquellos en que ha sido necesario extenderlas a órganos vecinos, la vejiga; se puede practicar o bien el tema de colostomía seca en la región de las fosa iliaca izquierda, en los uréteres agotados en la piel, o bien implantar estas al colon unos cuantos centímetros por encima de la boca de la colostomía con los cuales se tiene el tipo de colos- 
tomía llamada húmeda que requiere el uso permanente de aparatos para la comodidad de estos, el sitio debe ser en la línea media cerca al ombligo. Nosotros en el caso que se presenta empleamos la colostomía seca y la implantación de los uréteres a la piel del abdomen. Aunque en muchos sitios se continúan usando los aparatos para colostomía, saco, bolsas, etc., sin embargo las ventajas indudables del método de las irrigaciones colónicas para el manejo cómodo y menos desagradable de las colostomías, se está imponiendo desde hace varios años. Evidentemente el hecho que los pacientes no necesiten cargar sacos atados al abdomen donde se colectan las heces fecales si no llevar una simple gasa que proteja delicada mucosa del roce de la ropa, justifica sobradamente el tiempo que pierden primero todos los días y luego cada tercer día practicándose sus irrigaciones colónicas, que luego le permiten estar limpios y libres de aparatos hasta el día, o dos días siguientes.

Los aparatos usados para la irrigación de la colostomía pueden ser de tres tipos diferentes. El set de irrigación de Binkley, que consiste en una copa transparente de plástico con una ventosa en el centro que permite el paso de una sonda No. 16 o 18, una sábana de caucho que se usa grande o pequeña, según el paciente haga su irrigación en la cama o en la silla, un cinturón elástico para mantener en posición, la copa plástica y una pequeña bolsa de caucho que, se usa mientras se hacen regulares los hábitos de los movimientos colónicos.

Si el paciente es ambulatorio, las irrigaciones se pueden hacer en un inodoro o en un asiento cerca de él.

Se une a la parte baja de la copa de sabanita de caucho de tal manera que ella pase por entre las piernas y llega al inodoro o al balde usado para colectar el producto de la irrigación. Después se llena un aparato semejante a los usados para enemas comunes y corrientes con agua, lubrica convenientemente la punta de las ondas y se introducen dentro del intersticio después se deja correr lenta y suavemente el agua a la cual se ha podido agregar un poquito de sal, se aprovecha la distensión que hace el agua para introducir un poco más el tubo, se deja correr el agua hasta que el paciente sienta la sensación de llenura del colon, entonces se retira la sonda y se deja salir el contenido colónico repitiendo hasta que salga claro, algunas veces hay que esperar un tiempo hasta de media hora, y hasta que el colon expulse toda la cantidad de líquido introducido y quede por lo tanto limpio y el paciente pueda quedar tranquilo hasta su nueva irrigación usando solamente un apósito de protección.

El segundo método el más sencillo y ha sido el usado por nosotros pues carecemos de un ejemplar del de Binkley, y es solamente una sonda rectal delgada que se introducen dentro del colon por ella se hace pasar agua bien con la ayuda de una jeringa grande un set usual de enema, y una riñonera para recoger el producto de la irrigación, tiene la desventaja que siempre se escapa un poco de líquido y se usa mayor cantidad de material para la limpieza del paciente que frecuentemente se salpica un poco y se moja de esos liquidos.

El tercer método consiste en hacer la irrigación con una jeringa, pero es menos seguro, requiere más experiencia en las manos del que lo hace, pues es más fácil de maltratar la mucosa con la punta o con la presión del líquido entrante.

La primera irrigación se suele hacer 5 o 6 días después de la operación, comenzándolas a hacer cuando el paciente se halla en cama, pues la sensación naturalmente no era para él, lo mismo que el trauma psíquico que seguramente decidirá el mejor que lo hallen reclinado en una cama y no sentado en una silla o en un baño. Posteriormente se le acostumbra hacerlo en la posición sentada, quizás más fácil y cómoda. Las soluciones usadas pueden ser simplemente agua, o salina débil y algunos usan soluciones muy débiles de permanganato de potasio. Nosotros hemos usado simples cantidades de agua.

La vasija que contiene el líquido que se ha de introducir no ha de estar en una superior a $50 \mathrm{~cm}$ para evitar la fuerte presión que mayores alturas ocasionaría y la brusquedad de la salida del agua de tubo y su presión sobre la delicada mucosa intestinal. Antes de comenzar la irrigación se debe dejar correr un 
poco de líquido para expulsar el aire que se halle en la luz de los tubos y también en los sitios de clima frío, calentar un poco la sonda que introduce dentro del colon. No debe empujarse fuertemente la sonda pues la punta puede causar erosiones de la mucosa principalmente cuando se halla una bolsa ciega que con frecuencia en estas circunstancias se forma en el intestino.

Cuando a la entrada del líquido se presentan calambres abdominales, se detiene la entrada del líquido hasta que ellos pasen y luego se continúa con la irrigación. Ella ha de hacerse paulatinamente hasta emplear varios litros para en ningún caso pasar de seis. Debe enseñársele al paciente a ayudar de alguna manera desde el principio a la aplicación de irrigaciones, explicar la razón de ella y sus ventajas, y ganar su confianza contestando honradamente sus preguntas, darle ánimo y hacerle caer en cuenta de que no es un inválido o ser inferior por el hecho de llevar colostomía que no tiene por qué alejarlo de su vida común de sociedad ni menos imposibilitarlo para ejercer los trabajos comunes y corrientes de cualquier ciudadano.

Las colostomías húmedas necesitan aparatos permanentes y en ningún caso se practicarán irrigaciones pues ellas ayudarían a provocar infecciones ascendentes en los uréteres. Tampoco es recomendable el uso de laxantes o purgantes.

La irrigación perineal tiene por objetos lavar las amplias superficies cruentas, arrojando los restos de tejidos necrosados, las secreciones y las cantidades de pus que se formen en ellas, y permitir una más rápida y limpia granulación. Hay tres maneras de practicarlas y los resultados son semejantes, usándose unas u otras a gusto de los cirujanos encargados. La primera es con el paciente acostado en la cama, con las rodillas separadas y en flexión, y una mano separando las nalgas y con la otra una jeringa grande de unos $100 \mathrm{~cm}$ se irriga suavemente la solución tibia de permanganato de potasio. La segunda es usando una vasija a más de $50 \mathrm{~cm}$ de altura por razones similares a las explicadas en las colostomías. La tercera es sentando al paciente en una bañera y dándole los comunes baños de asiento por un tiempo de más o menos 20 minutos. En todo ellos se permite escurrir la cantidad remanente de la superficie quede más o menos seca y luego aplicar amplios apósitos que cubran la región. Nosotros empleamos el primer método, las irrigaciones fueron practicadas por tres meses hasta tanto la cicatrización fue completa. Generalmente se hicieron diarias, pero algunas veces en el día la rapidez de la granulación y de cicatrización de la zona perineal depende casi exclusivamente de buenos cuidados que se le tengan, de la buena técnica y frecuencia en practicar las irrigaciones y claro está, del estado general de paciente.

\section{HISTORIA CLÍNICA}

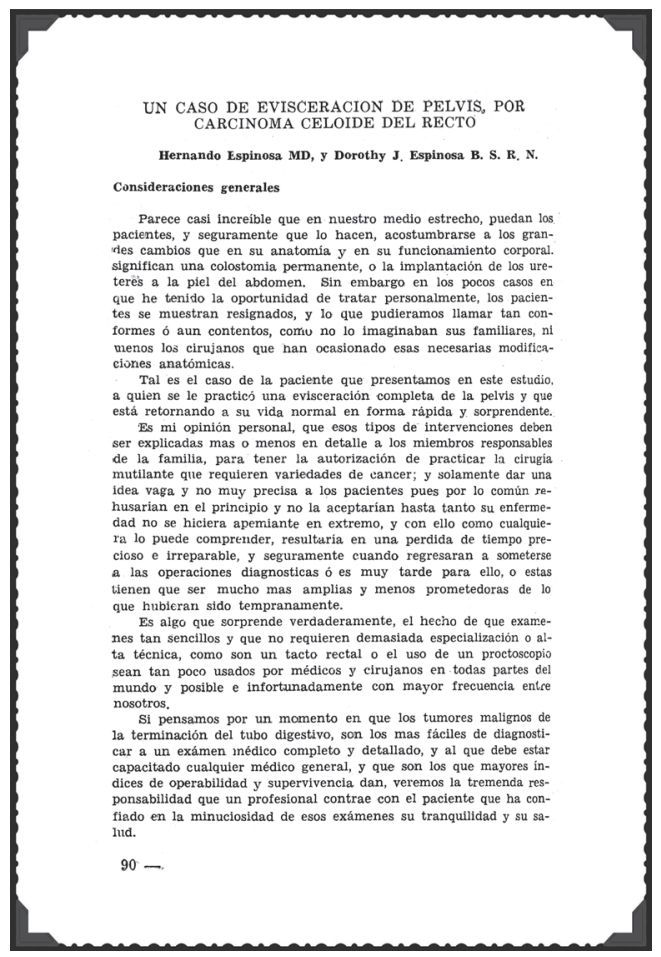

Paciente G. M. de B. que consulta en noviembre de 1952, de 47 años de edad, recientemente viuda quejándose que hace poco menos de seis meses notó la presencia de sangre roja mezclada con las deposiciones, en cantidad variable, pero en general ha ido aumentando con el tiempo. Presencia de abundante moco. Pus y sensación de peso en el recto y como si algo estuviera comprimiéndolo, a la vez que la impresión de defecación incompleta. Cambios notables en los hábitos de la defecación, pues habiendo sido toda la vida de correctos movimientos, des- 
de hace tiempo largo, aun antes de notar la sangre comenzó a sufrir de estreñimiento al cual no le dio importancia, pero ha aumentado y últimamente las deposiciones se han vuelto diarreicas. En síntesis sus defecaciones se han transformado en una mezcla líquida de heces, sangre, moco y pus, de muy mal olor y con sensación de peso en el recto y de defecación incompleta.

Fue tratada hace unos tres meses antes de su primera consulta en alguna clínica particular, donde laparotomizada y una histerectomía parcial le fue practicada, la paciente dice que con el diagnóstico de fibroma o retroversión uterina. Después de la intervención la paciente se sintió peor, sus síntomas aumentaron, se notó pálida y dice que ha perdido considerable cantidad de peso.

\section{EXAMEN FÍSICO}

Paciente de sexo femenino, acercándose a la quinta década de la vida, pálida, deprimida y con la sensación de sentirse gravemente enferma.

Cuatro embarazos a término, no tuvo abortos. No ha vuelto a reglar desde hace cinco meses. Aparato respiratorio y cardiovascular normal. Radiografías de tórax no muestran anormalidad. Abdomen blando, depresible, cicatriz infraumbilical mediana. Fosa iliaca izquierda empastada, dolorosa a la palpación. Ginecológico: vagina con presencia de flujo blancuzco, pared vaginal posteriormente empastada, rechazada junto con el cuello uterino hacia adelante en forma muy notable, por una masa casi inmóvil del tamaño de una naranja pequeña y francamente colocada por detrás de estos órganos. Tacto rectal: a unos $7 \mathrm{~cm}$ al ano se aprecia una gran masa que oblitera casi totalmente la luz del intestino, difícilmente movilizable, y al hacer los tactos vaginal y rectal combinado se aprecia el tumor firmemente adherido a la pared vaginal. Sale el dedo lleno de materias fecales líquidas con sangre y moco y de muy mal olor. La proctoscopia confirma los datos tomados por el tacto rectal. Al examen cistocópico, y este tiene que mantenerse en una posición casi vertical, es imposible localizar los orificios ureterales y hay disminución de la capacidad vesical.

\section{DIAGNÓSTICO CLÍNICO}

Carcinoma del recto, propagados a los tejidos vecinos, vagina, cuello uterino y muy posiblemente a la vejiga y uréteres. Como tratamiento se considera la posibilidad de una operación de Miles combinada con una resección abdomino-pélvica de los ganglios o la misma más extirpación de la vagina y el cuello uterino, o en último caso una evisceración completa de pelvis o una colostomía paliativa.

\section{OPERACIÓN}

Después de cuidadosa preparación, referente al estado general, pues el paciente solo tenía dos millones de glóbulos rojos y $60 \%$ de hemoglobina, y a su colon localmente se intervino el día 4 de diciembre de 1952, cirujanos Doctores Hernando Espinosa, Víctor Piñeros y Francisco Carmona. Anestesia, raquídea pentotal y éter. Presión arterial al comenzar la intervención 110-70 incisión transversa amplia cortando los rectos del abdomen y ligando las epigástricas. Se hace cuidadoso examen del abdomen, hígado sin metástasis, ganglios grandes a lo largo de las iliacas. El tumor ocupa prácticamente la totalidad de la pelvis y es imposible encontrar a pesar de buscarlo largamente un sitio de clivaje entre el tumor y la vejiga. Se abre el peritoneo posterior por detrás, pues hacia delante se halla firmemente adherido y en considerable extensión al tumor se liga la hemorroidal superior y se separa el sigmoide. Se ligan las arterias iliacas internas y se hace la disección de los ganglios a lo largo de las iliacas, siendo necesario resecar la vena derecha por las firmes adherencias de unos ganglios colocados por detrás de ella, se practica luego la separación entre el tumor y el sacro al cual lo unen densas adherencias. Luego se hace la separación de la pared anterior de la vejiga encontrándose una tremenda vascularización principalmente en su parte inferior.

La vascularización hacia los lados de la masa es también extraordinariamente rica y la intervención para la separación se hace lenta y difícil, siendo necesario adelantar paso a paso y a costa de gran cantidad de ligaduras y buen número de transfusiones de sangre para mantener al paciente en estado satisfac- 
torio. Se cortó el colon envolviendo la parte distal en un guante de caucho y con el cabo proximal una colostomía en el ángulo izquierdo de la herida. Se implantaron los uréteres cerca al ombligo haciendo dos pequeñas contraberturas un poco por encima de la incisión. Se dejó el colon cerrado con una pinza y envuelta en gasa vaselinada, y los uréteres caracterizados con sondas No. 8.

Las condiciones de la paciente son excelentes, pulso de 90 , y la presión arterial 10 puntos por encima de la tomada al comienzo de la operación, pero habiéndose empleado en la fase abdominal casi seis horas y no disponiéndose de más sangre, en ese momento se resuelve dejar la vía perineal para corto tiempo después.

En diciembre 9 de 1952, usándose pentotal éter, se intervino por la vía perineal, cirujanos doctores Hernando Espinosa, Diego Espinosa y Francisco Carmona, anestesista Agustín Garcés, enfermera instrumentadora Laura Soto. Se practicó la parte perineal correspondiente a una operación de Miles, se prolongó la disección hacia los lados y hacia delante, separando la vagina de los tejidos cercanos, luego se extirpó gran parte de la vulva para poder avanzar hasta debajo del pubis y separar las uniones remanentes de la vejiga y extirpar todos los órganos en masa.

No hubo sitios sangrantes que valieran la pena, se empaquetó por un corto tiempo con compresas calientes y luego se hizo una cuidadosa revisión. Se aplicó una sabanita de caucho que se empaquetó con gasa vaselinada. Se abrió la colostemia y se dejó una sonda de caucho gruesa. Terminada la intervención, la paciente se haya en magníficas condiciones, pulso de 100 por minuto lleno y fuerte, presión arterial de 130-90.

El postoperatorio sin complicaciones, se usó dieta baja en residuos, solamente se formó un pequeño absceso alrededor de la abocadara del uréter izquierdo.

No hubo ningún trastorno circulatorio del miembro inferior derecho.

La herida cicatrizó por primera intensión, y las suturas se retiraron a los 8 días. A los 10 comenzaron las irrigaciones colónicas, empleando una simple sonda aplicada en el colon, se acostumbró primero a practicarlas diariamente y luego cada tercer día, fue necesario suspenderla por un corto tiempo la paciente presentó bandeja con abundante moco, pero luego volvió todo a la normalidad. Se practicaron irrigaciones perineales con soluciones débiles y tibias de permanganato de potasio diariamente, hasta tanto la granulación y cierre de la enorme superficie cruenta se hizo, actualmente está completamente cicatrizada la zona y no causa molestia.

Por varias semanas se mantuvieron cateterizados los uréteres, pero en tres ocasiones se presentaron fiebre de tipo urinoso, que cedieron rápidamente a los antibióticos de aspecto amplio. Se consiguió un equipo mediano de los llamados "Singer cup" pero no fue bien tolerado por la paciente, por lo cual se nos ocurrió cortar el hongo de una sonda de pezzer grande y se aplicó a la piel alrededor de los uréteres fijándola con cemento a prueba de humedad marca Davel, con lo cual se ha obtenido un excelente resultado pues no ocupa casi ningún volumen y se ha logrado que funcionen sin despegarse hasta por una semana, cuando ello sucede se retiran, se limpia la piel y se aplica nuevamente después de limpiar cuidadosamente las partículas de cemento que queden adheridas a la sonda. Para ayudar a la buena fijación de los hongos de las sondas empleamos un aro metálico a través del cual sale el tubo y se fija a la piel con esparadrapos.

A los cuatros meses después de operada la paciente se halla en excelentes condiciones físicas, sus glóbulos rojos por encima de 4 millones y la hemoglobina en $75 \%$ a pesar de las incomodidades lógicas de las ureterostomías y las menores de sus colostomías la paciente se haya muy conforme y puede decirse que contenta con el resultado de su intervención, sale a la calle y viene semanalmente al hospital, $25 \mathrm{~km}$ de distancia, para chequeo y revisión y sin que ninguna persona que la vea pueda adivinar los profundos cambios que en su anatomía se han practicado.

El examen anatomopatológico practicado por el doctor Haroldo Calvo reporta la presencia de un carcinoma de tipo coloide y la presencia de numerosas células tumorales en la luz de los vasos sanguíneos. 